\title{
Welchen Lohn verdient die Medizin?
}

\section{Samia Hurst}

Institut Ethique Histoire Humanités (iEH2), Medizinische Fakultät der Universität Genf

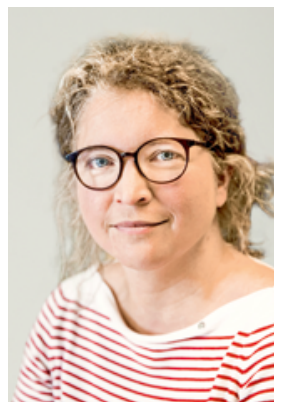

«Wie sollen Ärzte künftig behandeln, wenn sie ihre Patienten nur noch 20 Minuten sehen?» Die Bezahlung der Medizin ist keine einfache Sache. In der Politik können wir beobachten, wie sehr die Entlohnung der Ärzte die Gemüter erhitzt, sobald das Thema zur Sprache kommt. Vom ethischen Standpunkt aus ist es eine mindestens ebenso heikle Frage. Denn schliesslich verdienen wir unser tägliches Brot mit dem Leid unserer Mitmenschen. «Fordere, was dir zusteht, so lange der Schmerz da ist», sagte ein Arzt im Mittelalter nach einem Zitat im British Medical Journal von 1908. «Nach der Genesung haftet dem Arzt ein schlechter Geruch an».

Dennoch müssen die Ärzte von etwas leben können. Denn wer wollte in einer Welt leben, in der die Medizin ausstirbt, weil wir mit ihr nicht mehr unseren Lebensunterhalt bestreiten können? Doch noch nie in der Geschichte wurde der finanzielle Gewinn der Ärzte ganz ohne Argwohn betrachtet. Immer wieder herrschte und herrscht eine ambivalente Haltung gegenüber dem Bild des reichen Arztes. Geht es ihm vielleicht mehr um sein Geld als um das Wohl der Patienten? Oder ist sein Wohlstand vielmehr ein Zeichen dafür, dass er ein besonders guter Arzt ist? Fehlt es ihm womöglich an Nächstenliebe (so stellte sich die Frage vor Einführung der Sozialversicherungen) und sorgt er nicht ausreichend für die Armen? Die Bezahlung der Medizin birgt gleichzeitig ein Risiko, denn so mancher Patient ist nicht in der Lage zu zahlen. Dieses zunächst nur individuelle Risiko wird zunehmend als kollektives Problem betrachtet. Die Gesellschaft an sich befürchtet heute, nicht mehr für die medizinische Versorgung aufkommen zu können. Was aber ist eine gerechte Entlohnung? In der Medizin wird nicht das Ergebnis vergütet, das viel zu sehr dem Zufall unterliegt. Aber was wird dann bezahlt? Wir zahlen für die Leistungen, und wir zahlen für die Zeit. Im DRG-System zahlen wir je nach Krankheit, in anderen Ländern werden die Ärzte pro Patient bezahlt. All diese Systeme bergen eigene Probleme. Sollte also die Konsultation als Leistung vergütet werden, die auf 20 Minuten begrenzt ist? Das bedeutet, dass wir einen Teil ehrenamtlich erbringen. Selbstverständlich sind wir darüber verärgert! Zumal sich die Begrenzung unverhältnismässig stark bei den Spezialisten auswir- ken wird, die mehr Zeit benötigen und deren Haupteinnahme sich aus der Zeitkomponente ergibt. Sie sind es nicht, die derzeit am besten verdienen, könnte man sagen. Als die Abrechnung nach Minuten eingeführt worden war, erzählten aber die Kolleginnen und Kollegen von einer anderen unangenehmen Situation. Indem sie jede Minute in Rechnung stellten, hatten sie den Eindruck, ihren Beruf nicht mehr im Dienste des Mitmenschen auszuüben, ihren Patienten nichts Eigenes mehr zu geben. Einige fühlten sich damit zutiefst unwohl.

Hierin zeigt sich etwas grundsätzlich Wahres. Nur selten besteht unser Lohn sowohl aus einer finanziellen Vergütung als auch aus Dankbarkeit. Wenn alles bezahlt ist, ist unsere Pflicht abgegolten. Und darin liegt sogar der Sinn der Bezahlung, denn sie macht eine Transaktion zu etwas Unpersönlichem, damit sie auch ohne jegliches gegenseitiges Vertrauen durchgeführt kann [1] Daher ist es nicht verwunderlich, dass in der Medizin, wo Vertrauen und die persönliche Beziehung unverzichtbar sind, Geld ein heikles Thema ist.

Hinter den wiederkehrenden Debatten verbergen sich also viele Fragen, die vermutlich niemals erschöpfend beantwortet werden können. Dennoch kann man die Sache auch von einer anderen Warte aus betrachten. Vergütungen sind immer auch Motivationsanreize. Was wollen wir also? Dass die Ärzte mehr Zeit mit ihren Patienten verbringen - oder weniger? Dass kranke Menschen häufiger zum Arzt gehen - oder seltener? Es ist bekannt, dass der Geldfaktor die Laufbahnentscheidung der jungen Ärztinnen und Ärzte beeinflusst. Wollen wir also mehr von den einen oder von den anderen Fachärzten?

Je nachdem, ob wir fragen: «Was ist eine gerechte Entlohnung?» oder «Welchen Effekt wollen wir erzielen?», werden die Antworten unterschiedlich ausfallen. Dennoch sind beide Fragen legitim. Die Debatten darüber sind schwierig, unbequem und unvermeidlich spannungsgeladen. Im Sinne unseres Gesundheitssystems sind sie jedoch unverzichtbar.

\footnotetext{
Literatur

1 Graeber D. Debt: the first 5000 years. New York: Melville House Publishing; 2011.
} 